\title{
Combination of thermal fundamentals and Deep Learning for infrastructure inspections from thermographic images. Preliminary results
}

\author{
by I. Garrido*, S. Lagüela**, Q. Fang*** and P. Arias*
}

* Applied Geotechnologies Research Group, Centro de Investigación en Tecnoloxías, Enerxía e Procesos Industriais (CINTECX), Universidade de Vigo, Campus Universitario Lagoas-Marcosende, 36310 Vigo, Spain, ivgarrido@uvigo.es; parias@uvigo.es

** TIDOP Research Group, EPS Ávila, University of Salamanca, Calle Hornos Caleros 50, 05003 Ávila, Spain, sulaguela@usal.es

*** Computer Vision and Systems Laboratory, Department of Electrical and Computer Engineering, Université Laval, 1065, av. de la Médecine, Québec, QC, G1V 0A6, Canada, qiang.fang.1@ulaval.ca

\begin{abstract}
The application of Deep Learning (DL) models using the measurements acquired by Non-Destructive Testing (NTD) tools as input data stands as a versatile solution for highly automated analysis. However, DL models using thermal images as input data are quite scarce when it comes to analysing defects in medium- and large-scale bodies. Therefore, this paper proposes the application of a thermal criterion and a DL model, Mask R-CNN, in thermal images acquired from different infrastructures with thermal bridges and moisture. The thermal criterion is first applied to the input data, showing its utility to improve DL models performance.
\end{abstract}

\section{Introduction}

The continued emergence of new methods and technologies for infrastructure inspections is an increasingly necessary requirement due to the continuous deterioration of man-made structures. In addition, the strong demand for indoor thermal comfort conditions and high energy savings to address climate change, is a real fact within the building field, especially in the most developed countries. Therefore, in order to be able to undertake preventive maintenance tasks to avoid deterioration and meet the previous requirements, the new tools and methodologies must focus on the most common and severe defects, including moisture and thermal bridges.

Through its different phase changes, moisture is able to deteriorate any type of infrastructure if this defect is not identified during its initial growth stage. From the oxidation in metallic infrastructures to efflorescence, cracking or detachment would be some of the deterioration phenomena moisture can cause. Furthermore, moisture can have a considerable impact on user health [1] due to the biological/mould formation and can also increase the energy demand [2] for heating/cooling in winter/summer due to the presence of moist materials. Moreover, thermal bridges are classified as the main causes of energy losses in buildings [3]. In addition, they can be sensitive parts of buildings by increasing the risk of moisture formation due to condensation as consequence of the decrease of the temperature of the interior surfaces in winter conditions [4].

On the other hand, Non-Destructive Testing (NDT) tools are currently the best techniques for infrastructure inspections because they neither damage nor disturb the structure under study. Focusing on the analysis of thermal bridges and moisture, several papers have achieved good results in different infrastructures using InfraRed Thermography (IRT) as NDT technique over the last years $[3,5,6]$. As advantages, IRT is a non-contact and operator-safe tool, in addition to enabling a high speed of inspection, exclusive application fields, and the possibility of post-processing the thermal images acquired [7]. Nevertheless, the automation of the interpretation of the thermal images is still a great challenge due to the low resolution of the infrared cameras in the market, the great dependence of the environmental conditions, the homogeneous heating that needs to be provided on the surface under study and the control of the different mechanisms of heat transfer [7, 8]. Few IRT papers have shown the possibility to automatically analyse thermal bridges [8,9] and moisture $[8,10,11]$ in different types of infrastructures by applying new thermal image post-processing algorithms.

Moreover, Deep Learning (DL) has made a remarkable progress with the continued emergence of new models since the beginning of the $21^{\text {st }}$ century [12]. Compared to other artificial intelligence learning methods, DL is making a great improvement in solving problems that were difficult to handle in the artificial intelligence research fields for many years. This is mainly due to the fact during the training of a DL model, each neuron represents a basic classifier and the neurons of a layer are shared by the neurons of the next layer as a module to build classifiers, and this modularization learns from the data used as input. As a consequence, there are several recent published papers that show the potential of the use of DL models applied to measurements obtained by different NDT techniques [13-15]. Regarding the most recent IRT works, Fang et al. [12], Duan et al. [16], Yousefi et al. [17] and Ali [18] proposed DL models applied to thermographic data to automatically identify various subsurface defects belonging to different types of small-scale materials. All of them shown good results, overcoming the disadvantages of IRT when it comes to automatically interpreting thermal images. 
Therefore, in this paper a DL model of supervised learning, Mask R-CNN, is used in combination with a thermal criterion for the automatic detection, classification and delimitation of thermal bridges and moisture from a thermographic dataset of different infrastructures (Section 2). The thermal criterion is first applied in order to improve the contrast of the defect areas (Section 3.1), which is based on the research lines performed in the above-cited IRT papers that developed automation methods for infrastructure inspection so far [8-11]. Then, the training and validation of the DL model is applied to the thermal images with the improved contrast in the defect areas (Section 3.2). With this, the second goal of the paper is to demonstrate how it is possible to improve the performance of DL models applied to thermographic data by combining them with thermal fundamentals (Section 4). Furthermore, the performance obtained with the above-cited automation IRT works [8-11] is also compared (Section 4), ending with some conclusions and discussions on future steps (Section 5).

\section{Dataset description}

The dataset used in this work consists of thermal images with thermal bridges and moisture affecting the surfaces of the structures previously detected by an operator. All of them are acquired with the same infrared camera (see specifications in Table 1).

Table 1. Specifications of the infrared camera used.

\begin{tabular}{cc}
\hline Sensor type & Uncooled focal plane array ( pbolometer) \\
Thermal image/pixels & $640(\mathrm{H}) \times 480(\mathrm{~V})$ \\
Resolution $\left({ }^{\circ} \mathrm{C}\right)$ & 0.1 \\
Accuracy & $\pm 2{ }^{\circ} \mathrm{C}$ or $\pm 2 \%$ of reading, whichever is greater \\
Spectral range $(\mu \mathrm{m})$ & 8 to 14 \\
\hline
\end{tabular}

The thermal images represent different parts of buildings, from both inside and outside, pillars of various bridges and different regions inside tunnels. It is noted that the environmental conditions at the time of acquisition of each thermal image are different among images, that in all the thermal images the emissivity value has been set at 1 and that the reflected temperature and atmospheric effects have been compensated. Besides, although the resolution of the infrared camera used is $640 \times 480$, a Region of Interest (ROI) in each thermal image has been chosen in order to cover only the defect areas of interest. Figure 1 shows some thermal images of the dataset in 8-bit format, placed next to their corresponding visible images and ROls. The 8-bit format conversion ( 0 to 255 integer pixel values) is performed as a preprocessing step in order to stretch each thermal image histogram. In this way, the contrast of the entire thermal image is improved with the application of the 8-bit format conversion, which facilitates the subsequent improvement of the contrast of the defect areas after the application of the thermal criterion and, consequently, the improvement of the DL performance.

(a)
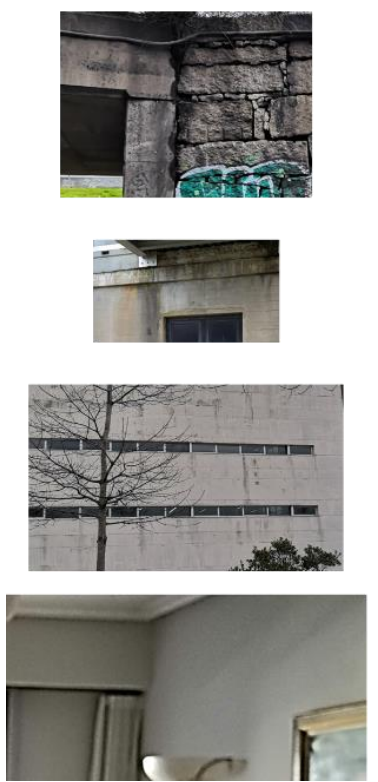

(b)
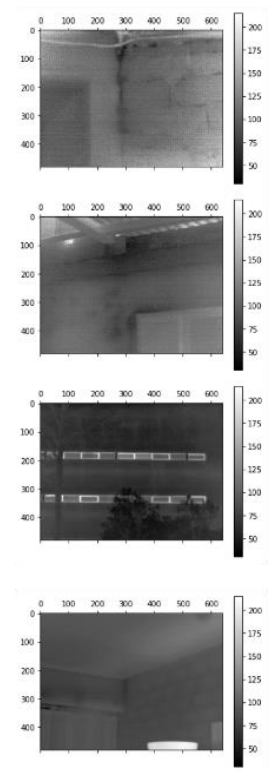

(c)

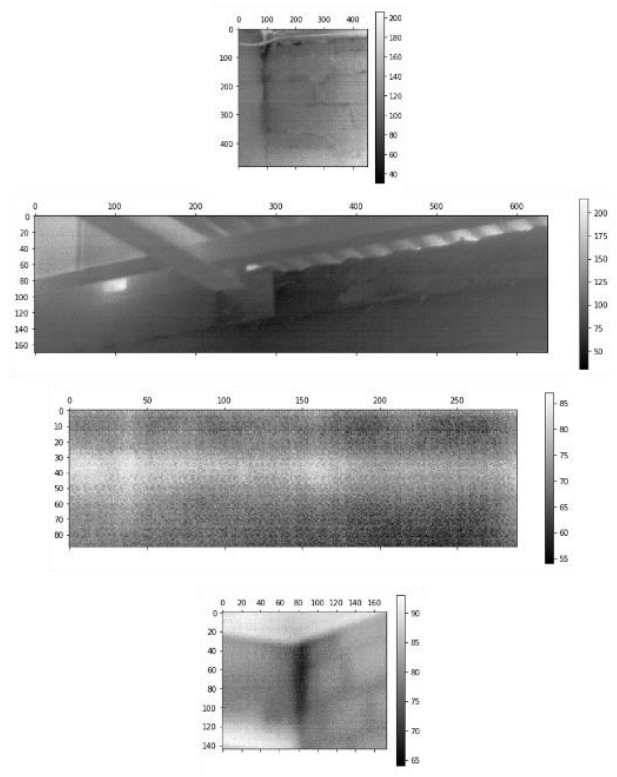

Fig. 1. Some thermal images of the dataset used in this work (b), placed next to their corresponding visible images (a) and ROls (c). The first two rows represent moisture, and the last two rows represent thermal bridges. 


\section{Methodology}

\section{1. $1^{\text {st }}$ Step: Thermal criterion application for defect areas contrast enhancement}

According to references [8-11], the thermal behaviour of a defect, thermally affecting the surface of the structure under study, results in a Gaussian temperature distribution. In short, the Gaussian bell of a defect will appear in the histogram of a thermal image if the thermal footprint of the defect appears on the surface of the infrastructure. Thus, this thermal criterion consists of identifying the regions in each thermal image of the dataset used (i.e., in each ROI chosen) that present Gaussian distributions, with the subsequent purpose of enhancing their contrasts.

For that, the Savitzky-Golay (SG) smoothing filter is first applied to the histogram of the ROI. This filter is based on the least-squares method, which main advantage is that it tends to preserve characteristics of the initial distribution (in this case the ROI histogram) such as relative maximum and minimum values while softening the intermediate values [19]. The output is a line that adjusts to the shape of the ROI histogram without taking noise into account and preserving the extremes of possible Gaussian bells, that is, preserving the relative minimum values. Figure 2 shows an example of the application of the SG smoothing filter on a ROI.
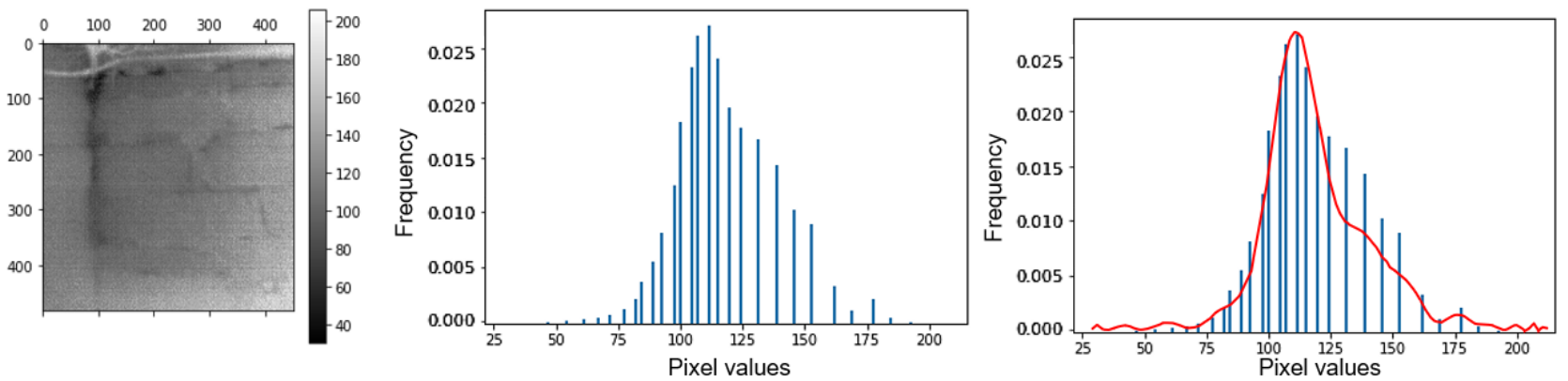

Fig. 2. Example of the SG smoothing filter application. From left to right: ROI, ROI histogram and SG smoothing filter output (red line).

Then, the relative minimum values of the SG smoothing filter output are identified. In this way, a mask is generated from the ROI for each interval between two consecutive relative minimum values, also taking into account the minimum and maximum pixel value of the ROI. It should be noted that a mask can contain different regions of the ROI that have their pixel values within the corresponding interval. Thus, a process of dilatation and a connecting method are applied to each mask. The first aims at grouping very close neighbours while the second aims at connecting components to label each region. The different regions are candidates for moisture or thermal bridge areas. Figures $3 a$ and $3 b$ show the corresponding result regarding the SG smoothing filter output from Figure 2.

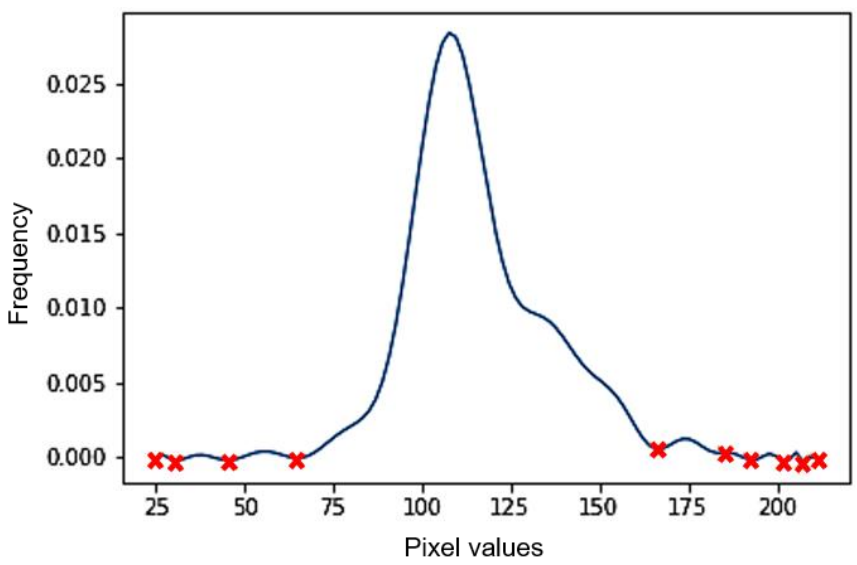

Fig. 3a. Relative minimum values identification (red crosses) regarding the SG smoothing filter output from Figure 2. 


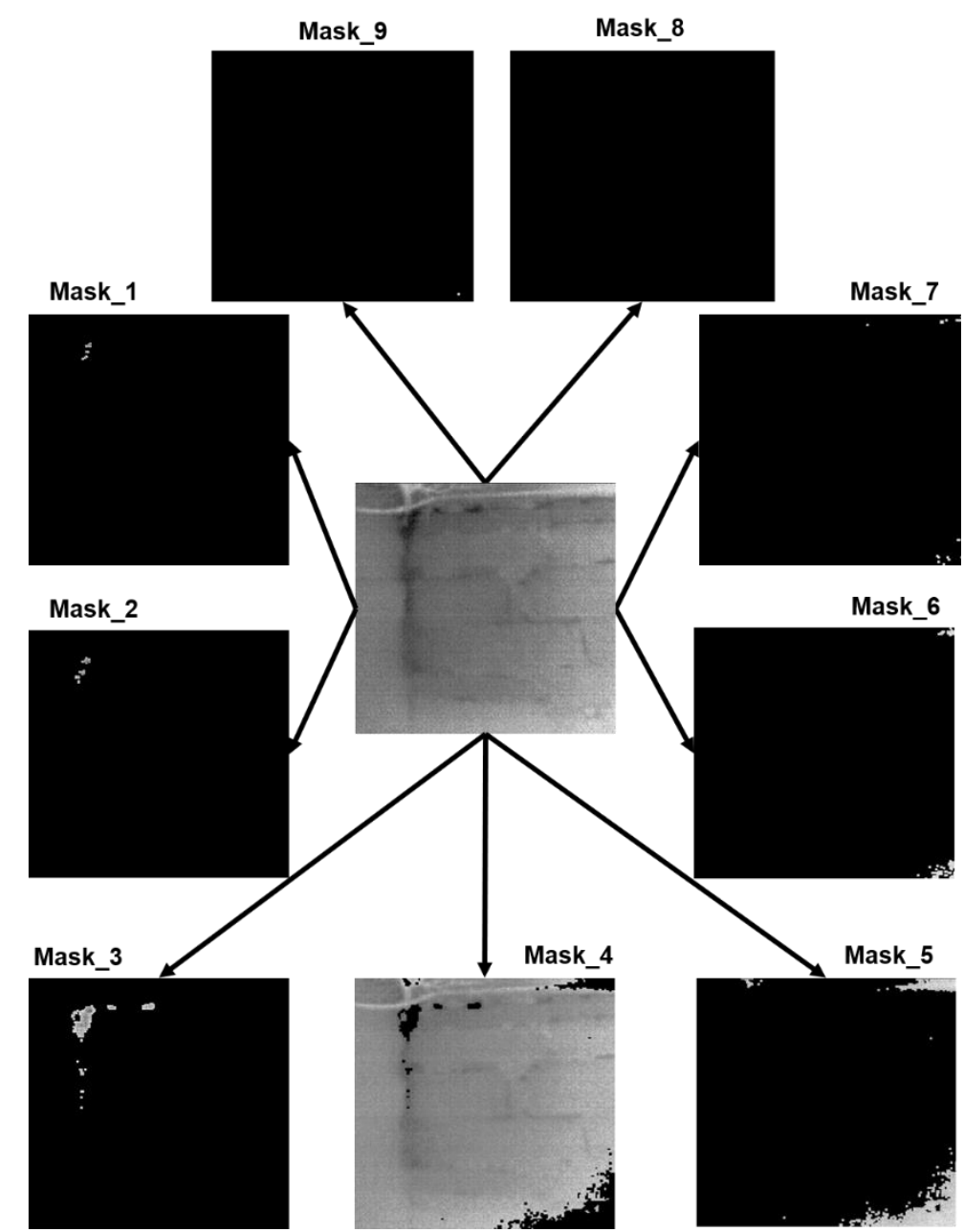

Fig. 3b. Candidates to moisture or thermal bridge areas (the non-dark zones of each mask) regarding the SG smoothing filter output from Figure 2.

Finally, the thermal criterion is applied to the candidates for moisture or thermal bridge areas. Specifically, the thermal criterion is based on the values of kurtosis and skew calculated from the pixel values of each candidate to be a defect area. The thermal criterion consists on subjecting the values of kurtosis and skew to a threshold interval. Thus, candidates that do not have a kurtosis and skew value between -2 and +2 are rejected as candidates because they do not present Gaussian distributions [10], i.e., they are not defect areas but noise or some artefact. Consequently, the rejected candidates are discarded from further processing.

After applying the thermal criterion, the Histogram Equalization (HE)-based contrast enhancement technique is applied to each accepted candidate. HE flattens and expands the dynamic range of the pixel values distribution of each accepted candidate by remapping the grey levels based on each candidate Probability Density Function (PDF), improving the contrast of the distribution of pixel values of each candidate [20]. With the purpose of maintaining the original relative differences in pixel values among the accepted candidates of the ROI, the distribution of pixel values of each accepted candidate is interpolated to maintain its original width, i.e., without the HE stretching. It should be noted that the unaltered areas of the surface of a structure that do not have excessive noise, shadows and reflections in the thermal image also present Gaussian distributions. i.e., would also be considered as accepted candidates. However, although these areas are not really defect areas, improving their contrasts by HE also improves the DL performance since the relative difference between healthy and unhealthy areas is increased.

Figure 4 shows the final result obtained with respect to the ROI used as an example in this section. 

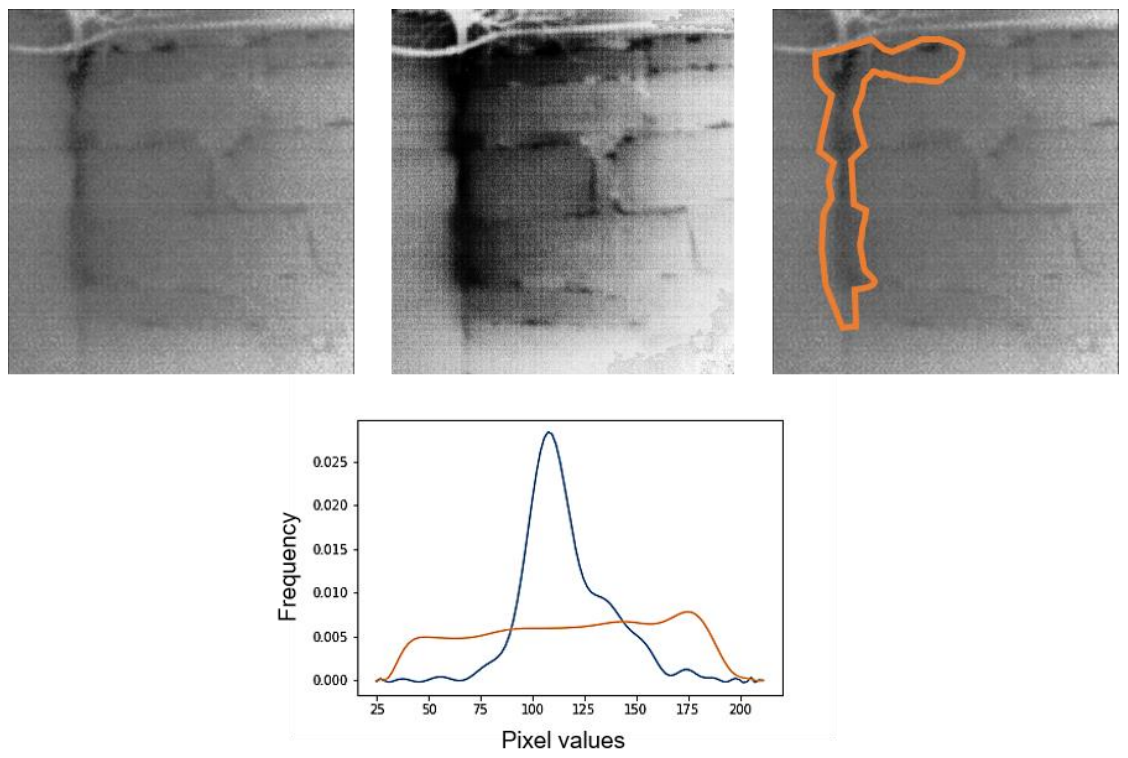

Fig. 4. Final result after thermal criterion and application of HE on the ROI used in this section. First row: initial $\mathrm{ROI}$ (left) and final result (centre), indicating the corresponding real defect areas by orange contours (right). Second row: blue and orange line belong to the SG smoothing filter output after applying to the ROI histogram, before and after thermal criterion and HE application, respectively.

\section{2. $2^{\text {nd }}$ Step: Learning process of the Deep Learning model}

Mask R-CNN [21] is the DL model used in this work. It is built on top of Faster R-CNN, which is another DL model that returns the bounding box coordinates and the class label for each object detected in the input image. However, Mask $\mathrm{R}-\mathrm{CNN}$, apart from returning the same outputs of Faster R-CNN, also returns the object mask being the latest state-of-theart framework for image segmentation tasks. Figure 5 represents the Mask R-CNN framework.

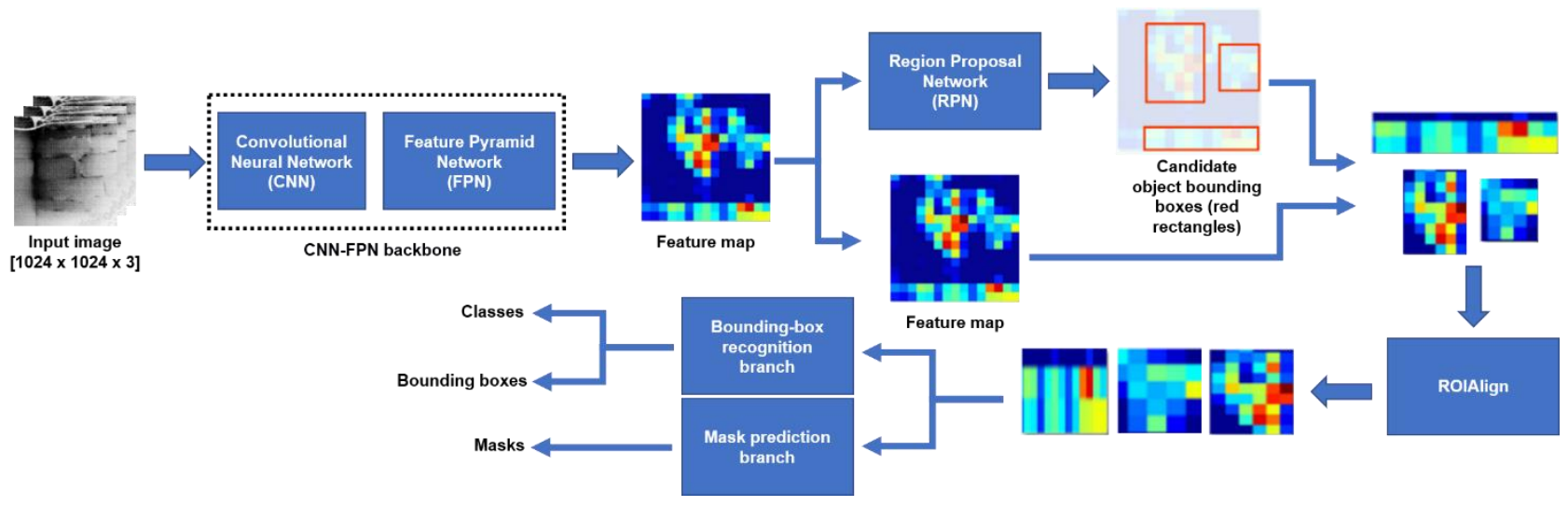

Fig. 5. The Mask R-CNN framework. Scheme modified from the reference [22].

As can be seen in Figure 5, the network architecture of Mask R-CNN is divided into: 1) a Convolutional Neural Network (CNN) architecture and a Feature Pyramid Network (FPN) used for feature extraction over the input image, 2) the Region Proposal Network (RPN), and 3) the network head for bounding-box recognition and mask prediction. The CNN architecture selected in this work is the ResNet [23] network of depth 101 layers, a common choice within the state-of-theart. As for the FPN, this network is a top-down architecture with lateral connections to build an in-network feature pyramid from a single-scale input. The size of the top-down layers used in this work is 256 , a common size value within the stateof-the-art. The reasons for using a ResNet-FPN backbone for feature extraction (feature map) over the input image are the excellent gains in both accuracy and speed [21]. The input image is the ROI after the thermal criterion application for the defect areas contrast enhancement, converting all the inputs to tensors of shape $(1024,1024,3)$ so that the DL model can learn from them. With the RPN, candidate object bounding boxes are proposed after scanning regions of the feature map. As last stage of Mask R-CNN, the bounding-box recognition and mask prediction branches are applied to each candidate object bounding box in order to provide the final outputs. It should be noted that 'ROIAlign' is designed for pixel- 
to-pixel alignment between network inputs and outputs, preserving the exact spatial location of each target object (in this case the moisture and thermal bridge areas).

For the Mask R-CNN training, 62 ROls are used, distributed in 33 with moisture and 29 with thermal bridges, having approximately $50 \%$ of each defect. Regarding the Mask R-CNN validation, 12 ROIs with moisture and 12 ROls with thermal bridges are used. Then, the dataset of this work is composed of 86 thermal images, $72 \%$ for training and $28 \%$ for validation. As for the labelling of each defect area, the VGG Image Annotator (VIA) software [24] is chosen given its simplicity and standalone manual annotation for images.

\section{Results}

The implementation of Mask R-CNN was on Python 3, Keras and TensorFlow, using the open source code from [25] as reference. The proposed DL model ran in a laptop using its CPU (Intel(R) Core(TM) i7-6700HQ CPU @ 2.60 GHz, RAM 16.00 GB, 64 bit Ubuntu 16.04 Operating System). With the objective of avoiding overfitting during the DL model learning process due to the small number of thermal images in the dataset, the following hyper-parameters are established:

- $\quad$ Batch Size $=1$

- $\quad$ Learning Rate $=0.001$

- $\quad$ Learning Momentum = 0.9

- Weight Decay $=0.1$

In addition, some data augmentation techniques are applied (horizontal flipping and image cropping) and the transfer learning method is used to perform the learning process due to the small size of the dataset. Specifically, the pretrained weights obtained by Mask R-CCN after learning the Microsoft COCO dataset [26] are used as initial weights. Moreover, all the layers of the DL model are trained since the ROls are not natural images like the images from the Microsoft COCO dataset.

Figure 6 shows the corresponding learning curves after the learning process. Specifically, the training and validation learning curves are represented to give an idea of the goodness of the model. In turn, Figure 7 also shows the corresponding learning curves after the learning process but using the input data without applying the thermal criterion proposed and, consequently, without improving the contrast of the defect areas.

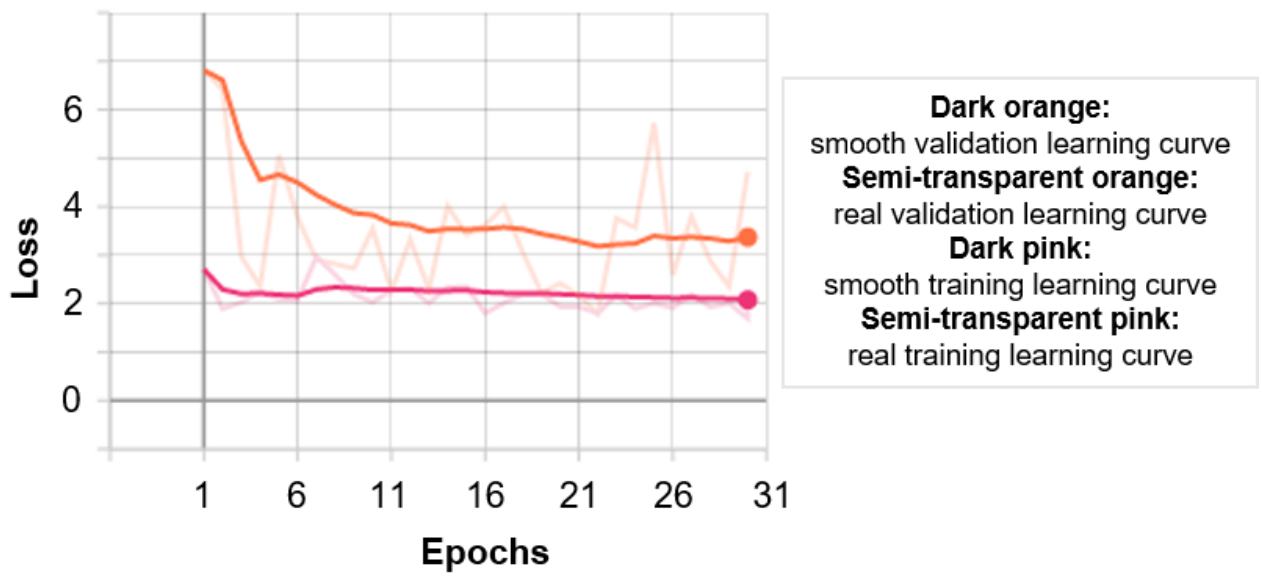

Fig. 6. Learning curves after the Mask R-CNN learning process, applying the thermal criterion in the input data.
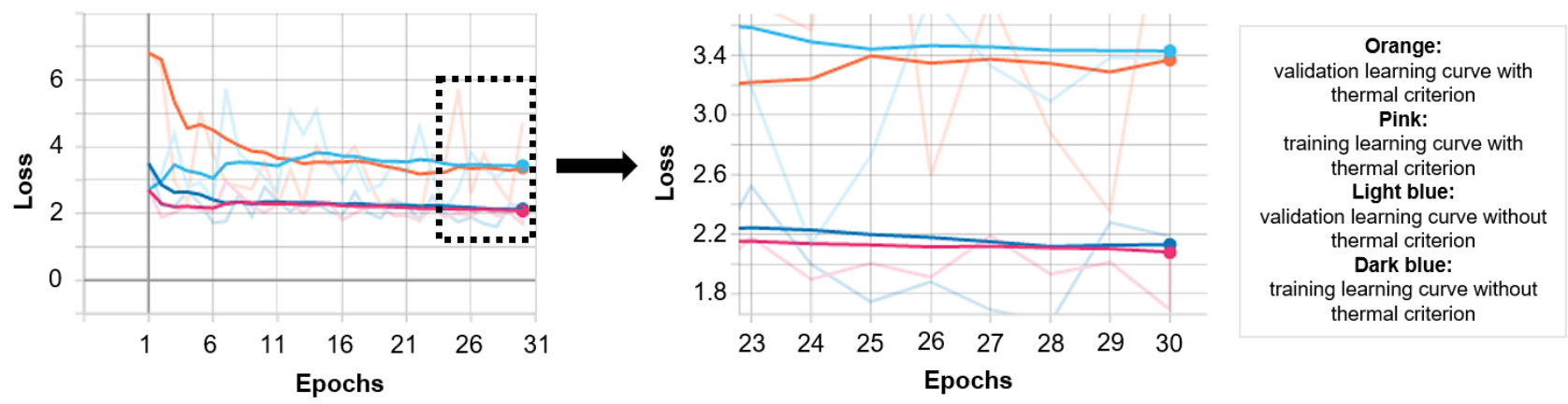

Fig. 7. Learning curves after the Mask R-CNN learning process, applying and not applying the thermal criterion in the input data. 
As can be seen in Figure 7, both learning processes stabilize after approximately 30 iterations (epochs), with the training and validation losses around 2.2 and 3.4, respectively, although slightly lower when applying the thermal criterion in the input data. Analysing quantitatively, the mean Average Precision (mAP) value obtained in each learning process by applying their weights after the $30^{\text {th }}$ iteration to the validation dataset is $18.75 \%$ using the thermal criterion and $17.13 \%$ without using the thermal criterion. With this, the usefulness of combining DL models with thermal fundamentals is demonstrated.

On the other hand, although the training and validation losses are still far from zero using the input data after applying the thermal criterion, the ROls that have obtained the best results have similar performance metric values compared to those obtained by the above-cited automation IRT works [8-11]. Specifically, the precision and recall values obtained by the above-cited automation IRT works range from around $60 \%$ to near $90 \%$. As for the best results of this work, these are shown in Figure 8, along with their corresponding precision and recall values and labelled defect areas (ground truth).

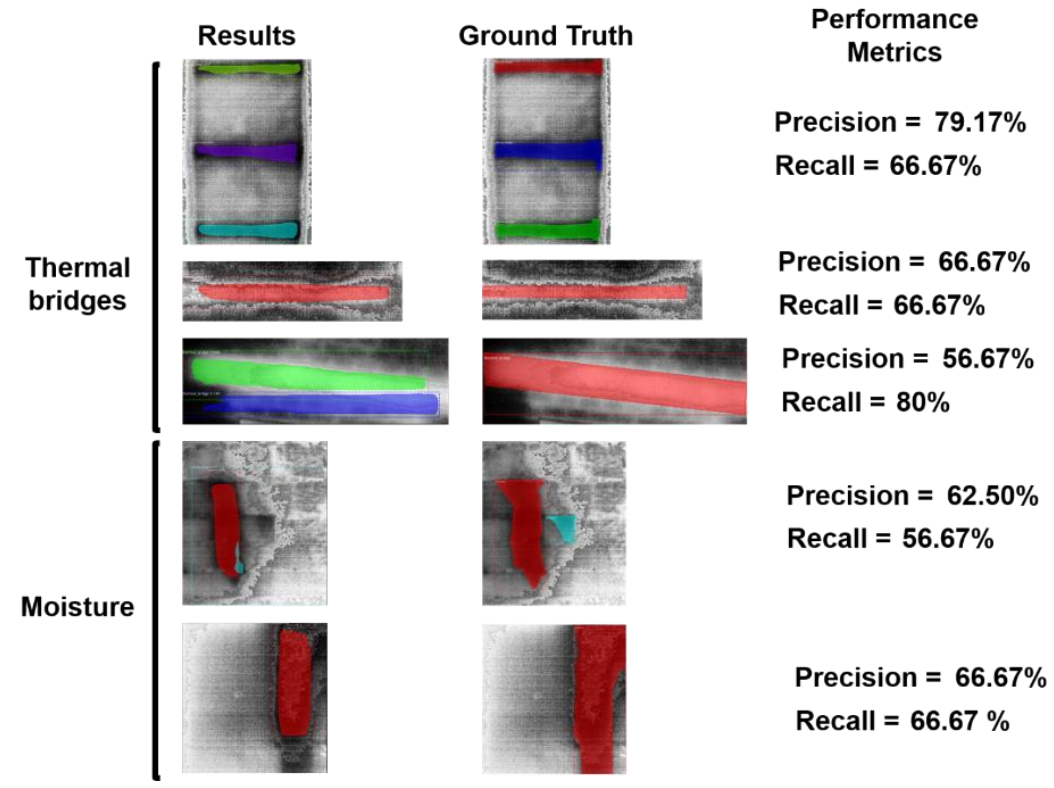

Fig. 8. Best results obtained.

\section{Conclusions and Future Perspectives}

Within the infrastructure inspection field, the automatic detection, classification and delimitation of thermal bridges and moisture has been performed in this work from a series of thermal images. For this purpose, thermal fundamentals and the latest state-of-the-art Deep Learning framework for image segmentation tasks have been combined to obtain an improvement in terms of detection, classification and delimitation with respect to using only the Deep Learning model. Although the results obtained are not optimal, this work takes the first step forward in the use of Deep Learning models for infrastructure inspection from thermographic data.

Future research will continue to improve the performance obtained, adding the same and/or different types of criteria and also using more real, and even simulated, thermal images in order to facilitate the increase in the amount of the input dataset. Likewise, it will be proposed to add to the analysis other types of defects that are common and severe in infrastructures, such as cracks, and to use other Deep Learning models.

\section{ACKNOWLEDGMENTS}

This research was funded by MINISTRY OF EDUCATION (GOVERNMENT OF SPAIN) through programs for human resources (FPU16/03950). Travel expenses were partially covered by the Travel Award sponsored by the open access journal Infrastructures published by MDPI.

\section{REFERENCES}

[1] Barreira, E.; Almeida, R.M.S.F.; L. Simões, M.; Rebelo, D. Quantitative Infrared Thermography to Evaluate the Humidification of Lightweight Concrete. Sensors 2020, 20, 1664, doi:10.3390/s20061664. 
[2] Edis, E.; Flores-Colen, I.; de Brito, J. Quasi-quantitative infrared thermographic detection of moisture variation in facades with adhered ceramic cladding using principal component analysis. Build. Environ. 2015, 94, 97-108, doi:10.1016/J.BUILDENV.2015.07.027.

[3] Garrido, I.; Lagüela, S.; Otero, R.; Arias, P. Thermographic methodologies used in infrastructure inspection: A review-Post-processing procedures. Appl. Energy 2020, 266, 114857.

[4] Ge, H.; Baba, F. Dynamic effect of thermal bridges on the energy performance of a low-rise residential building Energy Build. 2015, 105, 106-118, doi:10.1016/j.enbuild.2015.07.023.

[5] Usamentiaga, R.; Venegas, P.; Guerediaga, J.; Vega, L.; Molleda, J.; Bulnes, FG. Infrared Thermography for Temperature Measurement and Non-Destructive Testing. Sensors 2014, 14, 12305-12348, doi:10.3390/s140712305.

[6] Kylili, A.; Fokaides, P.A.; Christou, P.; Kalogirou, S.A. Infrared thermography (IRT) applications for building diagnostics: A review. Appl. Energy 2014, 134, 531-549, doi:10.1016/j.apenergy.2014.08.005.

[7] Madruga, F.J.; Sfarra, S.; Perilli, S.; Pivarčiová, E.; López-Higuera, J.M. Measuring the Water Content in Wood Using Step-Heating Thermography and Speckle Patterns-Preliminary Results. Sensors 2020, 20, 316, doi:10.3390/s20010316.

[8] Garrido, I.; Lagüela, S.; Arias, P. Autonomous thermography: towards the automatic detection and classification of building pathologies. In 14th Quantitative Infrared Thermography Conference; Berlin, Germany, 2018; pp. 359368.

[9] Garrido, I.; Lagüela, S.; Arias, P.; Balado, J. Thermal-based analysis for the automatic detection and characterization of thermal bridges in buildings. Energy Build. 2018, 158, 1358-1367, doi:10.1016/J.ENBUILD.2017.11.031.

[10] Garrido, I.; Lagüela, S.; Sfarra, S.; Madruga, F.J.; Arias, P. Automatic detection of moistures in different construction materials from thermographic images. J. Therm. Anal. Calorim. 2019, 1-20, doi:10.1007/s10973019-08264-y.

[11] Garrido, I.; Lagüela, S.; Sfarra, S.; Arias, P. Development of Thermal Principles for the Automation of the Thermographic Monitoring of Cultural Heritage. Sensors 2020, 20, 3392, doi:10.3390/s20123392.

[12] Fang, Q.; Nguyen, B.D.; Ibarra Castanedo, C.; Duan, Y.; Maldague, X. Defects detection in infrared thermography by deep learning algorithm. In Thermosense: Thermal Infrared Applications XLII; Oswald-Tranta, B., Zalameda, J.N., Eds.; SPIE, 2020; Vol. 11409, p. 31.

[13] Yu, X.; Tang, L.; Wu, X.; Lu, H. Nondestructive freshness discriminating of shrimp using visible/near-infrared hyperspectral imaging technique and deep learning algorithm. Food Anal. Methods 2018, 11, 768-780, doi:10.1007/s12161-017-1050-8.

[14] Riid, A.; Lõuk, R.; Pihlak, R.; Tepljakov, A.; Vassiljeva, K. Pavement Distress Detection with Deep Learning Using the Orthoframes Acquired by a Mobile Mapping System. Appl. Sci. 2019, 9, 4829, doi:10.3390/app9224829.

[15] Soilán, M.; Riveiro, B.; Balado, J.; Arias, P. Comparison of heuristic and deep learning-based methods for ground classification from aerial point clouds. Int. J. Digit. Earth 2019, doi:10.1080/17538947.2019.1663948.

[16] Duan, Y.; Liu, S.; Hu, C.; Hu, J.; Zhang, H.; Yan, Y.; Tao, N.; Zhang, C.; Maldague, X.; Fang, Q.; Ibarra-Castanedo, C.; Chen, D.; Li, X.; Meng, J. Automated defect classification in infrared thermography based on a neural network. NDT E Int. 2019, 107, 102147, doi:10.1016/j.ndteint.2019.102147.

[17] Yousefi, B.; Kalhor, D.; Usamentiaga, R.; Lei, L.; Ibarra-Castanedo, C.; Maldague, X. Application of Deep Learning in Infrared Non-Destructive Testing. In 2018 Quantitative InfraRed Thermography; QIRT Council: Berlin, Germany, 2018.

[18] Ali, R. Deep learning-and infrared thermography-based subsurface damage detection in a steel bridge; 2019.

[19] de Oliveira, M.; Araujo, N.; da Silva, R.; da Silva, T.; Epaarachchi, J. Use of Savitzky-Golay Filter for Performances Improvement of SHM Systems Based on Neural Networks and Distributed PZT Sensors. Sensors 2018, 18, 152, doi:10.3390/s18010152.

[20] Singh, K.; Vishwakarma, D.K.; Walia, G.S.; Kapoor, R. Contrast enhancement via texture region based histogram equalization. J. Mod. Opt. 2016, 63, 1444-1450, doi:10.1080/09500340.2016.1154194.

[21] He, K.; Gkioxari, G.; Dollár, P.; Girshick, R. Mask R-CNN. IEEE Trans. Pattern Anal. Mach. Intell. 2020, 42, 386397, doi:10.1109/TPAMI.2018.2844175.

[22] Taming the Hyper-Parameters of Mask RCNN - Analytics Vidhya - Medium Available online: https://medium.com/analytics-vidhya/taming-the-hyper-parameters-of-mask-rcnn-3742cb3f0e1b (accessed on Jun 19, 2020).

[23] He, K.; Zhang, X.; Ren, S.; Sun, J. Deep residual learning for image recognition. In Proceedings of the IEEE Computer Society Conference on Computer Vision and Pattern Recognition; IEEE Computer Society, 2016 ; Vol. 2016-December, pp. 770-778.

[24] Dutta, A.; Zisserman, A. The VIA Annotation Software for Images, Audio and Video. MM 2019 - Proc. 27th ACM Int. Conf. Multimed. 2019, 2276-2279, doi:10.1145/3343031.3350535.

[25] Mask_RCNN/samples/balloon at master · matterport/Mask_RCNN . GitHub Available online: https://github.com/matterport/Mask_RCNN/tree/master/samples/balloon (accessed on Jun 19, 2020).

[26] Lin, T.-Y.; Maire, M.; Belongie, S.; Bourdev, L.; Girshick, R.; Hays, J.; Perona, P.; Ramanan, D.; Zitnick, C.L.; Dolí, P. Microsoft COCO: Common Objects in Context. 